\title{
Condition-dependent expression of virulence in a trypanosome infecting bumblebees
}

\author{
M. J. F. BROWN, R. LOOSLI, P. SCHMID-HEMPEL \\ ETH Zürich, Experimental Ecology, ETH-Zentrum NW, CH-8092 Zürich, Switzerland
}

Corresponding author: M. J. F. Brown, ETH Zürich, Experimental Ecology, ETH-

Zentrum NW, CH-8092 Zürich, Switzerland. Tel: (+41) 1632 6935, Fax: (+41) 1632

1271, E-mail: brown@eco.umnw.ethz.ch 


\begin{abstract}
Parasite virulence affects both the temporal dynamics of host-parasite relationships and the degree to which parasites regulate host populations. If hosts can compensate for parasitism, then parasites may exhibit condition-dependent virulence, with high virulence being seen only when the host is under conditions of stress. Despite their usually low level of virulence, theory suggests that such parasites may still affect host population dynamics. We tested whether a trypanosome intestinal parasite of bumblebees, Crithidia bombi, expresses condition-dependent virulence. Hosts were infected with the parasite and then kept under either favourable or starvation (stressed) conditions. Under favourable conditions the infection caused no mortality, while when hosts were starved the infection increased the host mortality rate by $50 \%$. In addition, we found a parasiterelated change in host resource allocation patterns. Infected bees invested relatively more resources into their fat body and less into their reproductive system than did non-infected bees. Whether this reallocation is parasite-driven, to enhance transmission, or a hostresponse to parasitism, remains unknown.
\end{abstract}




\section{Introduction}

Parasite virulence, or pathogenicity, is a measure of the impact of a parasite on the fitness of its host. Together with host resistance, virulence largely determines how a host and its parasites interact, and how the the dynamics of the two interacting populations unfold (Lenski and May 1994). While some parasites are highly virulent, resulting in high mortality of infected hosts, many parasites have much more subtle effects (Ewald 1983). In such cases, compensation by the host, perhaps by enhanced resource consumption, may be obscuring the true cost of parasitism. Consequently, the major effects of parasitism will only appear when the host is under stress. Nevertheless, such parasites can still play an important role in regulating host populations (Anderson and May 1981).

An increase in parasite-induced effects with stressful conditions is probably a widespread phenomenon (e.g., Jokela (1999)) and consequently has many implications for the study of host-parasite interactions, both in the field and the laboratory. However, few data exist where increased host mortality under stressful conditions can be causally linked to parasitism (but see Schaub (1989), Jaenike (1995)). Trypanosome parasites of insects provide an opportunity to examine variation in pathogenesis with environmental stress. Generally, insect trypanosomes exhibit low levels of pathogenicity under conditions that are favourable to the host (Schaub 1994). However, a few studies suggest that, when environmental conditions for the host deteriorate, trypanosome infections do in fact impose a mortality cost (Vargas and Zeledon 1985, Schaub and Loesch 1989, Arnqvist and Mäki 1990). Insect trypanosomes are common and widespread (Schaub 1994) and thus, if condition-dependent virulence is a common phenomenon, may play a hitherto unrecognised role in regulating host populations. Here, we use a bumblebee-trypanosome 
host-parasite system to investigate whether trypanosome parasites increase host-mortality under stress conditions.

Bombus terrestris L. (Hymenoptera, Apidae) is an annual, eusocial insect. Queens found colonies in the spring, and workers forage for nectar and pollen from these colonies until mid- to late-summer. Reproductives (gynes and males) are produced at the end of the colony life-cycle, and after mating queens enter hibernation. Crithidia bombi (Trypanosomatidae, Zoomastigophorea, Lipa and Triggiani (1980)) is a prevalent parasite of Bombus spp. (Shykoff and Schmid-Hempel 1991a). Transmission occurs between colonies via flowers (Durrer and Schmid-Hempel 1994), and within colonies by contact with infected material, and occurs by ingestion of cells that are shed in the faeces of infected bees. Colonies usually contract an infection within two weeks of the start of foraging activity (Imhoof and Schmid-Hempel 1999). Infection by C. bombi has a number of subtle, pathogenic effects, including reduced ovary growth and slower colony growth at the start of the season (Shykoff and Schmid-Hempel 1991b). In addition, allopatric infections have been shown to increase host mortality rates, suggesting local adaptation between the parasite and its host (Imhoof and Schmid-Hempel 1998). A remarkable observation in this system is the occurrence of strong host-parasite genotypegenotype interactions, suggestive of strong coevolutionary dynamics (Schmid-Hempel and Schmid-Hempel 1993, Schmid-Hempel et al. 1999). However, as yet there is little evidence that $C$. bombi is sufficiently virulent to explain the genetic interactions between parasite strains and host colonies (Shykoff and Schmid-Hempel 1991c, Shykoff and Schmid-Hempel 1991d). Here we examine the effects of sympatric infections on their 
individual bumblebee hosts under both favourable and resource-stressed conditions for the host.

\section{Materials and methods}

\section{(1) Favourable conditions}

We collected queens of B. terrestris emerging from hibernation in spring 1996 in areas around Zurich, Switzerland. The queens were allowed to start a colony in the laboratory, which colonies were then kept under standard conditions $\left(20^{\circ} \mathrm{C}\right.$, ca. $60 \%$ r.H., food ad libitum) for their entire life cycle. Emerging queens may naturally be infected by $C$. bombi, and this infection is then passed on to the colony's workers. Some queens and their colonies typically remain uninfected under laboratory conditions.

For this first experiment (conducted in 1996), we used workers from a total of 15 non-infected colonies (the test colonies), and C. bombi extracted from 3 infected colonies. Thus, the $C$. bombi strains used for this experiment were sympatric with the experimental bees. Workers from the non-infected colonies were removed daily as callows from the nest, i.e., within 24 hrs after hatching, individually marked with numbered tags (Opalithplättchen ${ }^{\circledR}$ ), and returned to their natal colony. Four days later, these workers were again removed from the colony, starved for 2 hours and fed with 15 $\mu \mathrm{l}$ of standard inoculum (5 $\mu \mathrm{l}$ of $C$. bombi cell culture according to treatment, diluted with $10 \mu \mathrm{l}$ of sterilised sugar water). The inoculum was prepared by mixing roughly equal amounts of $C$. bombi cells from the 3 infected colonies that served as sources for the parasite. The workers were then kept individually in plastic boxes $(12 \times 10 \times 7 \mathrm{~cm})$ for 4 weeks (this period was chosen mostly because of the low mortality) and fed with 
sugar water (Api Invert ${ }^{\circledR}$, diluted 1:3) and pollen ad libitum. All workers were freezekilled after 4 weeks and stored in a freezer $\left(-20^{\circ} \mathrm{C}\right)$ until inspection.

We used 8 workers each per cell of the experimental design, i.e., per test colony and treatment. They were allocated at random to treatments: “infection” by the standard inoculum, or “non-infection” by feeding the equivalent amount of culture medium and sugar water without C. bombi. Worker body size was measured as the length of the radial cell in the right wing (mm) and did not differ between treatments. After the end of the experiment, the amount of fat was measured according to Ellers (1996). For this, the abdomen was dried at $70^{\circ} \mathrm{C}$ for 3 days and then weighed with a precision balance. The abdomen was then placed in $2 \mathrm{ml}$ of ether for 24 hrs to extract the fat. After rinsing with fresh ether, the abdomen was again dried for 3 days and weighed again. The amount of fat is indicated by the difference between these two measures. The relative fat content is defined as the ratio of amount of fat (mg) and body size of the worker (mm). At the same time, ovariole sizes were measured for workers of the first 9 out of 15 colonies. After removal, the abdomen was placed in Ringer solution. The 8 ovarioles were then carefully removed from the abdominal cavity and the lengths of the 3 largest ovarioles were measured under a stereo microscope (25x magnification). The ratio of the average of these 3 measurements $(\mathrm{mm})$ and body size $(\mathrm{mm})$ was used as relative ovariole size for the analyses. In addition to measuring ovariole sizes and fat contents, the survival of infected and non-infected workers over the four-week period was also noted. In some cases, measurements of ovarioles, fat, or cell counts in the faeces were not successful. Hence, sample sizes deviate slightly among groups. 


\section{(2) Stress experiment}

This experiment was run in a similar fashion. B. terrestris colonies were the offspring of colonies produced by queens caught in spring 1999 around Frauenfeld, Switzerland.

Rearing conditions were as described above. The C. bombi inoculum for this experiment was derived from 10 naturally infected colonies and, as with the previous experiment, the parasite strains were sympatric with the colonies used for infection.

Workers from 3 colonies of B. terrestris (colonies A31, A60, and L54) were used for the experiment (which was conducted in 2000). On day 0 of the experiment we removed, at random, approximately 50 workers from each colony. These workers were then kept in groups of approximately 25 in plastic boxes $(17 \times 13 \times 9 \mathrm{~cm})$ lined with cat litter, with ad libitum pollen and sugar water. On day 3 of the experiment workers were starved for 3 to 4 hrs, and were randomly assigned to the control and treatment groups. This random assignment prevented systematic differences between groups in worker size and age, which might have confounded the experimental manipulation. Control bees were fed with $10 \mu$ l of sugar water solution, while treatment bees were fed with $10 \mu \mathrm{l}$ of the $C$. bombi inoculum. Workers were then placed in groups of 25, according to colony and treatment, in plastic boxes (as described above) for a further 7 days to allow the $C$. bombi infection to become established (Schmid-Hempel and Schmid-Hempel 1993). On day 10 of the experiment we removed all food and the bees were allowed to starve. For each bee we recorded the time from the initiation of starvation to death. Dead bees were dissected blind with respect to treatment and we recorded intensity of C. bombi infection (cell counts from the rectum), ovary development (the mean length of the 6 terminal oocytes), body size (mean length of the radial cells of the forewings), and fat body size. As before, 
relative fat content is the ratio of fat body (mg) and body size (mm), and the relative ovariole size is the ratio of ovariole size $(\mathrm{mm})$ and body size $(\mathrm{mm})$.

All statistical analyses for both experiments were done with SPSS 6.1, with variables transformed as necessary to normalise variances. To account for differences among colonies, the residuals of relative ovariole size and relative fat content were extracted from an ANOVA with colony as a random block effect. These residuals were used in all further analyses.

\section{Results}

\section{(1) Favourable conditions}

A total of 240 workers from 15 colonies could be used in the experiment. Ten workers died within an hour of the start of the experiment (mortality was not biased between treatments) and were excluded from the analysis, leaving a total of 230 individuals. Of those, only 12 subsequently died during the experiment, with a non-significant difference of seven deaths in the infected group (mortality: $5.9 \%, n=118$ ) and five in the noninfected group (4.5\%, $n=112$ ). Therefore, no obvious mortality difference resulted from the experimental infection under favourable conditions, even though individual lifespan was longer than the expected lifespan in the field (e.g., Goldblatt and Fell 1987, Schmid-Hempel and Heeb 1991).

A MANOVA tested the effect of treatment group (infected, non-infected) on the dependent variables ovariole size and fat content (Tables 1, 2a). The combination of relative sizes of ovarioles and fat content was significantly affected by colony identity interacting with treatment, but, surprisingly, not by treatment alone. Relative ovariole 
size was smaller in infected as compared to non-infected workers. However, treatment had no significant effect in univariate tests. Fat content was higher on average in infected animals but not signficantly so. Colony identity affected ovariole size and fat content, while the colony-treatment interaction only affected the former. Most interestingly, the relationship between ovariole size and fat contents was changed by the infection, as shown by a significant interaction term in an ANCOVA with residual fat as the covariate and treatment as the fixed effect (Fig. 1a). The change suggests that the infection leads to a re-allocation of resources away from the ovarioles to the fat body. No meaningful relationship between infection intensity, body size, fat contents or ovariole size and life span could be calculated as almost all bees survived to the end of the experiment and were then freeze-killed.
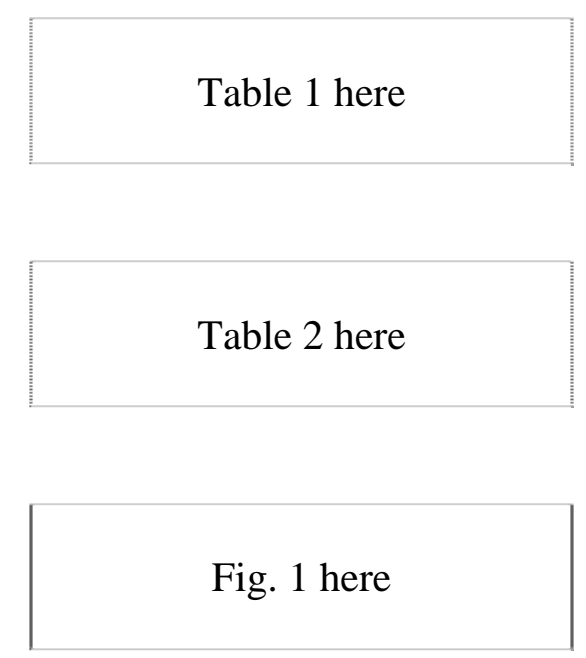

\section{(2) Stressed conditions}

17 out of 27, 20 out of 26, and 23 out of 24 treatment bees in colonies A31, A60, and L54 respectively, developed a C. bombi infection. All uninfected treatment bees were 
excluded from further analyses. All control bees remained free of C. bombi (25, 25, and 26 control bees for colonies A31, A60, and L54, respectively).

Crithidia bombi significantly reduced the survival probability of worker bees under these stressed conditions (Table 3, Fig. 2). We used a Cox regression analysis with forward selection to analyse the survival data. Survival time was the dependent variable, and colony, treatment, and the colony-by-treatment interaction were presented as candidate variables. Treatment and colony were coded using indicator and deviance coding, respectively, with the control treatment and colony A31 set as reference categories (to obtain statistics for colony A31, the analysis was repeated using colony L54 as the reference category). The final Cox regression model contained treatment, colony, and the colony-by-treatment interaction as significant predictors of survival. The main result was that inoculation with C. bombi increased mortality 1.5 times over that in the control bees. Colonies differed in their overall pattern of survival, as shown by the significant colony effect; bees from colonies A31, A60 and L54 suffered mortality rates $70 \%, 160 \%$ and $80 \%$ of the overall effect, respectively, although only for the first two colonies were these differences in mortality statistically significant. There was a significant interaction between treatment and colony, indicating that colonies differed in their susceptibility to C. bombi. Again, colony A60 had a significantly higher mortality rate, whilst colony L54 had a significantly lower mortality rate when compared to the overall effect.

Table 3 here 
Fig. 2 here

Despite variation in infection intensity among infected bees, there was no relation between intensity of infection and survival for either the combined data set or each colony individually (Spearman's Rank Correlation: All comparisons $P>0.1$ ). There was also no effect of colony on infection intensity (Kruskal-Wallis 1-way ANOVA, $\chi^{2}=1.0650$, D.F. $=2, P=0.5871)$. Finally, there was no relation between the intensity of infection and worker size (Spearman’s Rank Correlation: All comparisons $P>0.1$ ).

The length of time a worker survived starvation was not related to its size. There was no correlation between survival and worker size within any of the colony*treatment combinations (Spearman's Rank Correlation: All comparisons $P>0.1$ ).

Similar to the result under favourable conditions, infection changed the relationship of fat contents and ovariole size and again in the same direction, although only marginally so (see ANCOVA term, $P=0.08$, in Fig. $1 \mathrm{~b}$ ). The MANOVA for relative ovariole size and fat content indicated a colony-by-treatment effect for the combined response, but no effect was seen in univariate tests (Table 2b). These effects were less dramatic than in the first experiment, probably because the time allowed for the infection to affect the bees in this second experiment was much shorter (10 days vs. 28 days). Finally, we combined the data from the experiments under favourable and stressed conditions in a single analysis. As with previous analyses, to remove the colony effect, residuals for relative ovariole size and fat content were used. An ANOVA for residual ovariole size, with residual fat content as the covariate, demonstrated the same pattern as above, i.e., a strong treatment-by-condition interaction, suggesting that the effect of the 
treatment (infected vs. non-infected) depended on the condition of the workers (favourable vs. stressed). No direct effect of treatment or condition was found (Table 4). Note that the results stay the same when the three "outliers" in Fig. 1 (residuals of relative fat contents $>0.75$ ) are excluded.

\section{Discussion}

Condition-dependent virulence should be observed when a host has the potential ability to compensate for the pathogenic effects of its parasite. We found that the intestinal parasite C. bombi dramatically increases mortality in its bumblebee host under starvation conditions. In contrast, under favourable conditions the host was able to compensate for this mortality cost. Our starvation protocol mimics the natural situation, where foraging can be interrupted by rain and cold weather resulting in a costly energy shortfall (Cartar and Dill 1991). Thus, our results suggest that $C$. bombi could have major negative effects on the growth and survival of bumblebee colonies. This may be especially true for smaller colonies at the beginning of the foraging season, when weather conditions are often adverse for prolonged periods of time.

Our results are in line with a number of studies (Vargas and Zeledon 1985, Schaub and Loesch 1989, Arnqvist and Mäki 1990, Jaenike and Benway 1995, Jokela et al. 1999), suggesting that condition-dependent mortality may be a relatively common phenomenon. However, it should be noted that in these studies mortality occurred at the scale of days to months, whereas in our experiment, starvation conditions only had to persist for a few hours for the cost of parasitism to become apparent (Fig. 2). This growing body of evidence suggests that normally low-virulence parasites may play a more important role 
in the dynamics of their host populations than has been assumed. Anderson and May (1981) suggested that, if microparasite pathogenicity increased under stressful conditions, then the microparasite could regulate the population dynamics of its host. If, as in their model, stressful conditions, e.g., reduction in food availability, are caused by increasing host population density, then such parasites will act as density-dependent regulators of host populations. However, our results also suggest that microparasites may impact host populations at low density, if external factors, e.g., adverse meteorological conditions, govern food availability.

Our favourable- and stressed-environment experiments were run in different years, with different host colonies and parasite strains. While the ideal design would be to run both experiments with one set of colonies and strains, this was not possible for practical reasons. However, we do not believe that this is a problem with respect to the conclusion that $C$. bombi exhibits condition-dependent virulence. Firstly, by using multiple strains and colonies in each experiment, we minimised the risk of basing conclusions on results from a single and potentially unrepresentative colony-strain interaction. Secondly, previous experience shows that the result of no mortality under favourable conditions can be easily repeated in any year and with any colony (Schmid-Hempel 1998 and references therein, PSH unpublished data). Finally, the controls within each experiment argue that condition-dependent virulence is indeed real, and not an experimental artefact.

The second striking result of this study is the parasite-related reallocation of host resources. In both experiments, we found that the trade-off in resource allocation between the fat body and the reproductive system of the host had a different relationship in the infected than in the non-infected bees. Variation between experiments in the degree of 
this difference suggests that reallocation patterns change over a time-period of a few days. Why might infected bees allocate more resources to their fat body than to their reproductive system? One possibility is that the parasite is manipulating host resource allocation for its own benefit. Bees with larger fat reserves may live longer and support larger parasite populations, thus enhancing the probability of horizontal transmission. Similarly, a reduction in relative ovary size forces the workers to express their reproductive interests by manipulating offspring sex ratio rather than through their own reproduction, resulting in higher production of new queens and thus enhancing the vertical transmission of the parasite (Shykoff and Schmid-Hempel 1991d). An alternative explanation is that changes in trade-off relationships are a host response to parasitism. The fat body is an essential component of the immune system in invertebrates (Hultmark 1993), and by reallocating resources to the fat body, bees may be better able to withstand and combat infections. Further work is required to distinguish between these explanations.

The mechanism by which C. bombi decreases host survival is unclear, but several possibilities suggest themselves. Firstly, $C$. bombi may reduce the gut resource content that is available to starving bees by direct ingestion and, as they cover the epithelium of the gut wall (Gorbunov 1987, Gorbunov 1996), by blocking ingestion by the bee. Secondly, infection may inhibit the utilisation of gut contents by actually destroying intestinal cells (e.g., Jensen et al. (1990)). Thirdly, C. bombi may induce the host immune system, and the energy required for this induction may be sufficient to reduce host lifespan when additional resources are withheld (Moret and Schmid-Hempel, unpublished data). 
The level of reproduction by a parasite is usually taken to be a correlate of virulence (Bull 1994). Consequently, we expected to find a relationship within infected groups between survival and infection intensity. Surprisingly, there was no correlation between these two factors, suggesting that virulence and parasite reproduction are decoupled. Further work is needed to determine what features of parasite dynamics, e.g., the time lag between infection and the production of transmission cells, correlate with virulence in this bumblebee-trypanosome system.

We would like to thank Yannick Moret and Jürgen Wiehn for useful discussion. Thanks to Kerstin Huck for help in data collection. The manuscript was greatly improved thanks to comments from Jukka Jokela and an anonymous reviewer. This work was supported by a grant from the Swiss National Science Foundation (nr. 31-49040.96 to PSH) and a grant from the European Union and the Swiss BBW (nr.95.0575 to PSH).

\section{References}

Anderson, R. M. and May, R. M. 1981. The population dynamics of microparasites and their invertebrate hosts. - Philosophical Transactions of the Royal Society of London (B) 291: 451-524.

Arnqvist, G. and Mäki, M. 1990. Infection rates and pathogenicity of trypanosomatid gut parasites in the water strider Gerris odontogaster (Zett.)(Heteroptera: Gerridae). Oecologia 84: 194-198.

Bull, J. J. 1994. Virulence. - Evolution 48: 1423-1437. 
Cartar, R. V. and Dill, L. M. 1991. Costs of energy shortfall for bumble bee colonies: Predation, social parasitism, and brood development. - Canadian Entomologist 123: 283-294.

Durrer, S. and Schmid-Hempel, P. 1994. Shared use of flowers leads to horizontal pathogen transmission. - Proceedings of the Royal Society of London Series B Biological Sciences 258: 299-302.

Ellers, J. 1996. Fat and eggs: an alternative method to measure the trade-off between survival and reproduction in insect parasitoids. - Netherlands Journal of Zoology 46: 227-235.

Ewald, P. E. 1983. Host-parasite relations, vectors, and the evolution of disease severity. - Annual Review of Ecology and Systematics 14: 465-485.

Goldblatt, J. W. and Fell, R. D. 1987. Adult longevity of workers of the bumblebees Bombus fervidus (F.) and Bombus pennsylvanicus (De Geer)(Hymenoptera: Apidae). Canadian Journal of Zoology 65:2349-2353.

Gorbunov, P. S. 1987. Endoparasitic flagellates of the genus Crithidia (Trypanosomatidae, Zoomastigophorea) from alimentary canal of bumblebees. Zoologicheskii Zhurnal 66: 1775-1780.

Gorbunov, P. S. 1996. Peculiarities of life cycle in flagellate Crithidia bombi (Protozoa, Trypanosomatidae). - Zoologicheskii Zhurnal 75: 803-810.

Hultmark, D. 1993. Immune reactions in Drosophila and other insects: a model for innate immunity. - Trends in Genetics 9: 178-183.

Imhoof, B. and Schmid-Hempel, P. 1998. Patterns of local adaptation of a protozoan parasite to its bumblebee host. - Oikos 82: 59-65. 
Imhoof, B. and Schmid-Hempel, P. 1999. Colony success of the bumble bee, Bombus terrestris, in relation to infections by two protozoan parasites, Crithidia bombi and Nosema bombi. - Insectes Sociaux 46: 233-238.

Jaenike, J. and Benway, H. 1995. Parasite-induced mortality in mycophagous Drosophila. - Ecology 76: 383-391.

Jensen, C., Schaub, G. A. and Molyneux, D. H. 1990. The effect of Blastocrithidia triatomae (Trypanosomatidae) on the midgut of the reduviid bug Triatoma infestans. Parasitology 100: 1-10.

Jokela, J., Lively, C. M., Taskinen, J. and Peters, A. D. 1999. Effect of starvation on parasite-induced mortality in a freshwater snail (Potamopyrgus antipodarum). Oecologia 119: 320-325.

Lenski, R. E. and May, R. M. 1994. The evolution of virulence in parasites and pathogens: reconciliation between two competing hypotheses. - Journal of theoretical biology 169: 253-265.

Lipa, J. J. and Triggiani, O. 1980. Crithidia bombi, sp. n., a flagellated parasite of a bumble-bee Bombus terrestris L. (Hymenoptera, Apidae). - Acta Protozoologica 27: 287-290.

Schaub, G. A. 1994. Pathogenicity of trypanosomatids on insects. - Parasitology Today 10: 463-468.

Schaub, G. A. and Loesch, P. 1989. Parasite/host interrelationships of the trypanosomatids Trypanosoma cruzi and Blastocrithidia triatomae and the reduviid bug Triatoma infestans: Influence of starvation of the bug. - Annals of Tropical Medicine and Parasitology 83: 215-224. 
Schmid-Hempel, P. 1998. Parasites in social insects. - Princeton Univ. Press.

Schmid-Hempel, P. and Heeb, D. 1991. Worker mortality and colony development in bumblebees, Bombus lucorum (L.)(Hymenoptera, Apidae). - Mitteilungen der Schweizerischen Entomologischen Gesellschaft 64: 93-108.

Schmid-Hempel, P., Puhr, K., Kruger, N., Reber, C. and Schmid-Hempel, R. 1999. Dynamic and genetic consequences of variation in horizontal transmission for a microparasitic infection. - Evolution 53: 426-434.

Schmid-Hempel, P. and Schmid-Hempel, R. 1993. Transmission of a pathogen in Bombus terrestris, with a note on division of labour in social insects. - Behavioral Ecology and Sociobiology 33: 319-327.

Shykoff, J. A. and Schmid-Hempel, P. 1991a. Incidence and effects of four parasites in natural populations in bumble bees in Switzerland. - Apidologie 22: 117-126.

Shykoff, J. A. and Schmid-Hempel, P. 1991b. Parasites delay worker reproduction in bumblebees: Consequences for eusociality. - Behavioral Ecology 2: 242-248.

Shykoff, J. A. and Schmid-Hempel, P. 1991c. Parasites and the advantage of genetic variability within social insect colonies. - Proceedings Of the Royal Society Of London Series B Biological Sciences 243: 55-58.

Shykoff, J. A. and Schmid-Hempel, P. 1991d. Genetic relatedness and eusociality: Parasite-mediated selection on the genetic composition of groups. - Behavioral Ecology and Sociobiology 28: 371-376.

Vargas, L. G. and Zeledon, R. 1985. Effect of fasting on Trypanosoma cruzi infection in Triatoma dimidiata (Hemiptera: Reduviidae). - Journal of Medical Entomology 22: 683. 
Table 1. Relative ovariole size and fat content in infected and non-infected bees under favourable conditions.

\begin{tabular}{lcc}
\hline \hline & Infected & Non-infected \\
& (S.E.)(n) & (S.E.)(n) \\
\hline$\underline{\text { Favourable conditions: }}$ & & \\
Relative ovariole size $(\mathrm{mm} / \mathrm{mm})$ & $0.539 \pm 0.024(68)$ & $0.598 \pm 0.028(61)$ \\
Relative fat content $(\mathrm{mg} / \mathrm{mm})$ & $0.613 \pm 0.031(67)$ & $0.553 \pm 0.022(61)$ \\
Stressed conditions: & & \\
Relative ovariole size $(\mathrm{mm} / \mathrm{mm})$ & $0.837 \pm 0.028(57)$ & $0.775 \pm 0.029(73)$ \\
Relative fat content $(\mathrm{mg} / \mathrm{mm})$ & $0.584 \pm 0.026(60)$ & $0.551 \pm 0.022(76)$ \\
\hline \hline
\end{tabular}


Table 2. MANOVA for dependent variables relative ovariole size and relative fat content with fixed factor treatment and random factor colony, for (a) favourable, (b) stressed condition. Observed values are given in Table 1; also see Fig.1.

(a) Favourable condition

\begin{tabular}{lrrrrrr}
\hline \hline Effect & Wilks $\lambda$ & $\begin{array}{r}\text { df } \\
\text { effect, error }\end{array}$ & F & P & \multicolumn{2}{r}{$\begin{array}{r}\text { Univariate F } \\
(P)\end{array}$} \\
& & & & & $\underline{\text { fat }}$ \\
& & & & & & \\
& & & & & & \\
Treat & 0.636 & 2,7 & 2.003 & 0.205 & 0.800 & 4.494 \\
& & & & & $(0.397)$ & $(0.067)$ \\
Colony & 0.587 & 16,218 & 4.157 & $<0.001$ & 5.156 & 3.359 \\
& & & & & $(<0.001)$ & $(0.002)$ \\
Treat * Colony & 0.789 & 16,218 & 1.712 & 0.046 & 2.700 & 0.610 \\
& & & & & $(0.009)$ & $(0.768)$ \\
\hline \hline
\end{tabular}

(b) Stressed condition

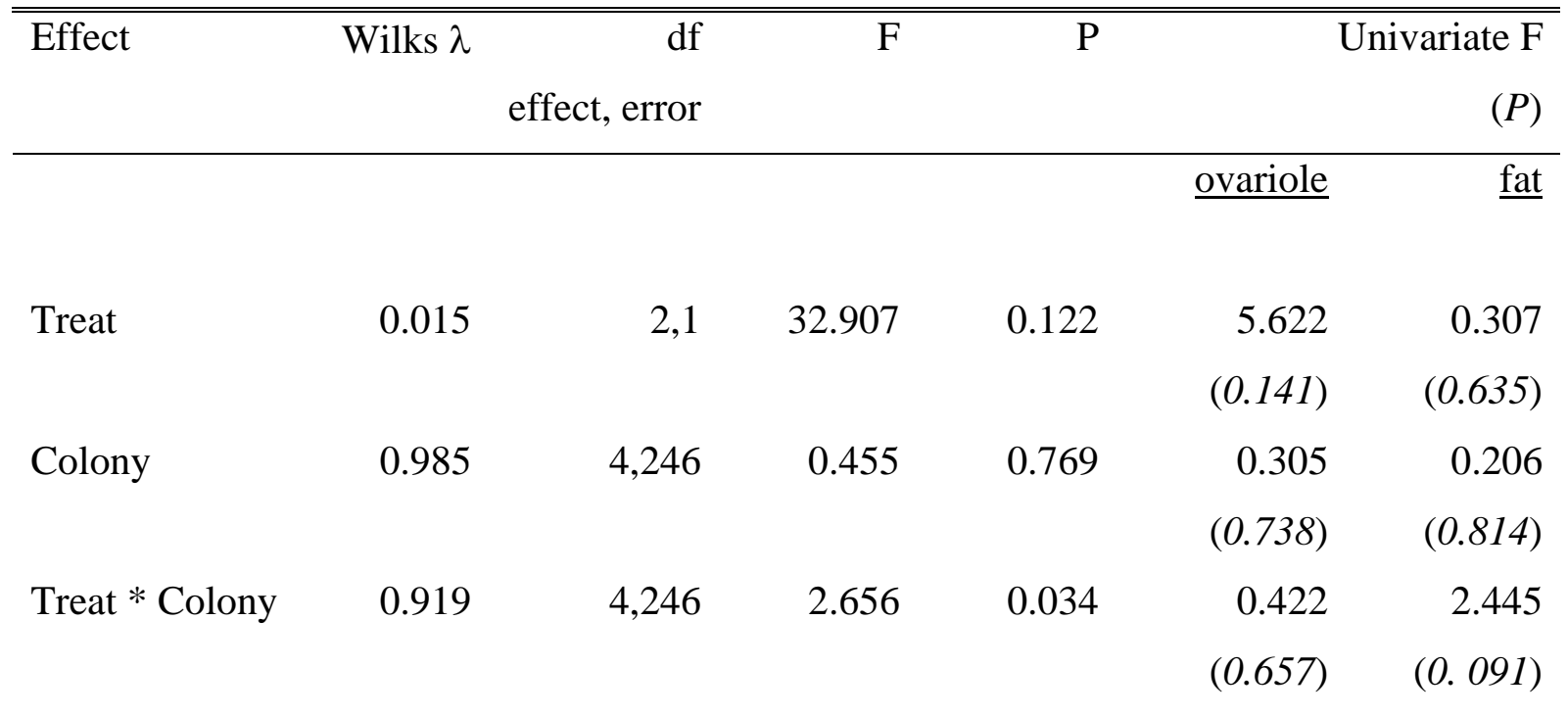


Table 3. Results of the Cox regression model for survival

\begin{tabular}{lllllll}
\hline Factors & $\mathrm{b}^{1}$ & S.E. $^{2}$ & Wald $^{3}$ & D.F. & $P^{4}$ & Odds $^{5}$ \\
\hline Crithidia vs. Control & 0.4428 & 0.1786 & 6.1453 & 1 & $\mathbf{0 . 0 1 3 2}$ & 1.5571 \\
Colony & & & 9.1205 & 2 & $\mathbf{0 . 0 1 0 5}$ & \\
A31 & & & & & & \\
A60 & -0.3626 & 0.1646 & 4.8524 & 1 & $\mathbf{0 . 0 2 7 6}$ & 0.6959 \\
L54 & 0.4815 & 0.1655 & 8.4682 & 1 & $\mathbf{0 . 0 0 3 6}$ & 1.6185 \\
Colony*Crithidia & -0.1190 & 0.1617 & 0.5410 & 1 & 0.4620 & 0.8879 \\
A31 (Crithidia) & & & & & & \\
A60 (Crithidia) & -0.1270 & 0.2542 & 0.2497 & 1 & 0.6173 & 0.8807 \\
L54 (Crithidia) & -0.6316 & 0.3530 & 6.2324 & 1 & $\mathbf{0 . 0 1 2 5}$ & 1.8807 \\
\hline \hline
\end{tabular}

${ }^{1}$ regression coefficient of the overall survival function for each factor

${ }^{2}$ standard error of the regression coefficient

${ }^{3}$ Wald statistic for each factor

${ }^{4}$ significance level of the Wald statistic

${ }^{5}$ odds ratio of survival for the factor relative to the control (exp(b))

${ }^{6}$ statistics obtained from setting colony L54 as the reference category 
Table 4. ANOVA of residuals for relative ovariole size after colony block effect is taken into account (combined data from both experiments). Residuals of fat content from colony block were entered as the covariate.

\begin{tabular}{lcccr}
\hline Source $^{1}$ & SS & $d f$ & $F$ & $P$ \\
\hline Treat & 0.01 & 1 & 0.21 & 0.644 \\
Condition & 0.01 & 1 & 0.03 & 0.853 \\
Treat * Condition & .24 & 1 & 6.34 & 0.012 \\
Covariate & & & & \\
Error & 1.56 & 1 & 42.03 & $<0.001$ \\
\hline \hline
\end{tabular}

1 Treat (infected / not-infected) and condition (favourable / stressed) as fixed effects.

2 Covariate: $\mathrm{t}=6.483 ; P<0.001$ 
Figure legends

Figure 1a. The relationship between the residuals of relative ovariole size and relative fat content for non-infected bees differs from that of infected bees under favourable conditions (ANCOVA of residuals from colony block effect: Treat * Residual-fat interaction: $\left.F_{1,125}=7.04, P=0.009\right) \mathbf{b}$. The relationship between the residuals of relative ovariole size and relative fat content for non-infected bees is marginally different from that of infected bees under stressed conditions (ANCOVA of residuals from colony block effect: Treat $*$ Residual-fat interaction: $\left.F_{1,125}=3.12, P=0.080\right)$. For statistics see Table 2. Note that both relationships hold whether or not the three "outliers" (residual fat > 0.75) are included in the analysis. The open diamonds and dashed lines represent data for the control bees, while the filled circles and solid lines represent data for the infected bees. Lines are best fit lines, and simply indicate the trend of the data points.

Figure 2. Infected bees have a higher mortality rate than non-infected bees. Each graph shows the data for one of the experimental colonies. The x-axis shows survival time and the y-axis the percentage of bees surviving in each group at each time point. The open diamonds represent data for the control bees, while the filled circles represent data for the infected bees. 
Fig. 1a

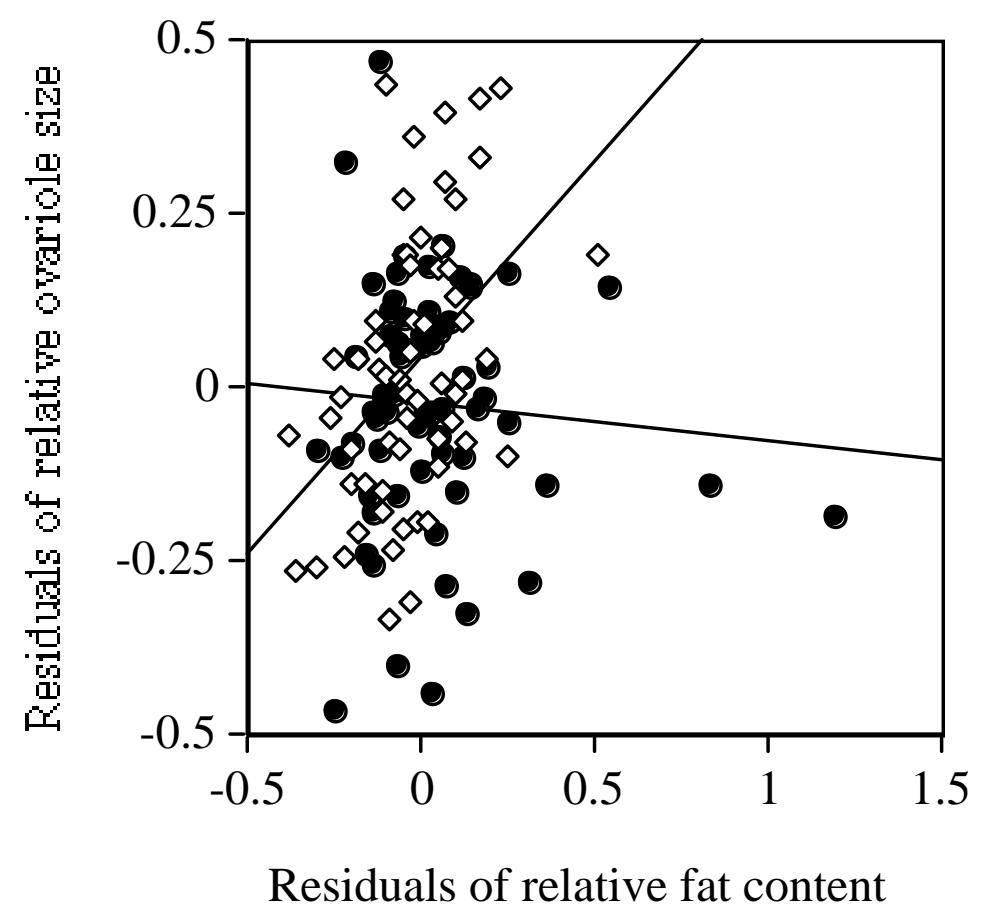

Fig. 1b

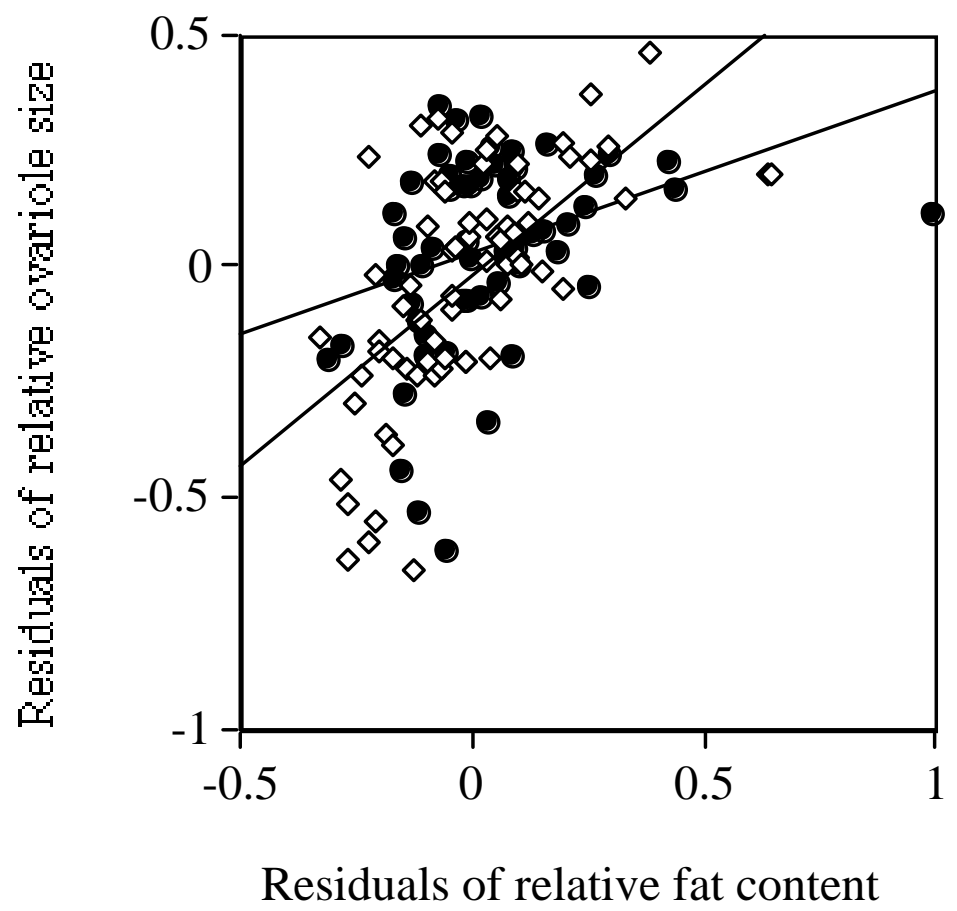


Fig. 2

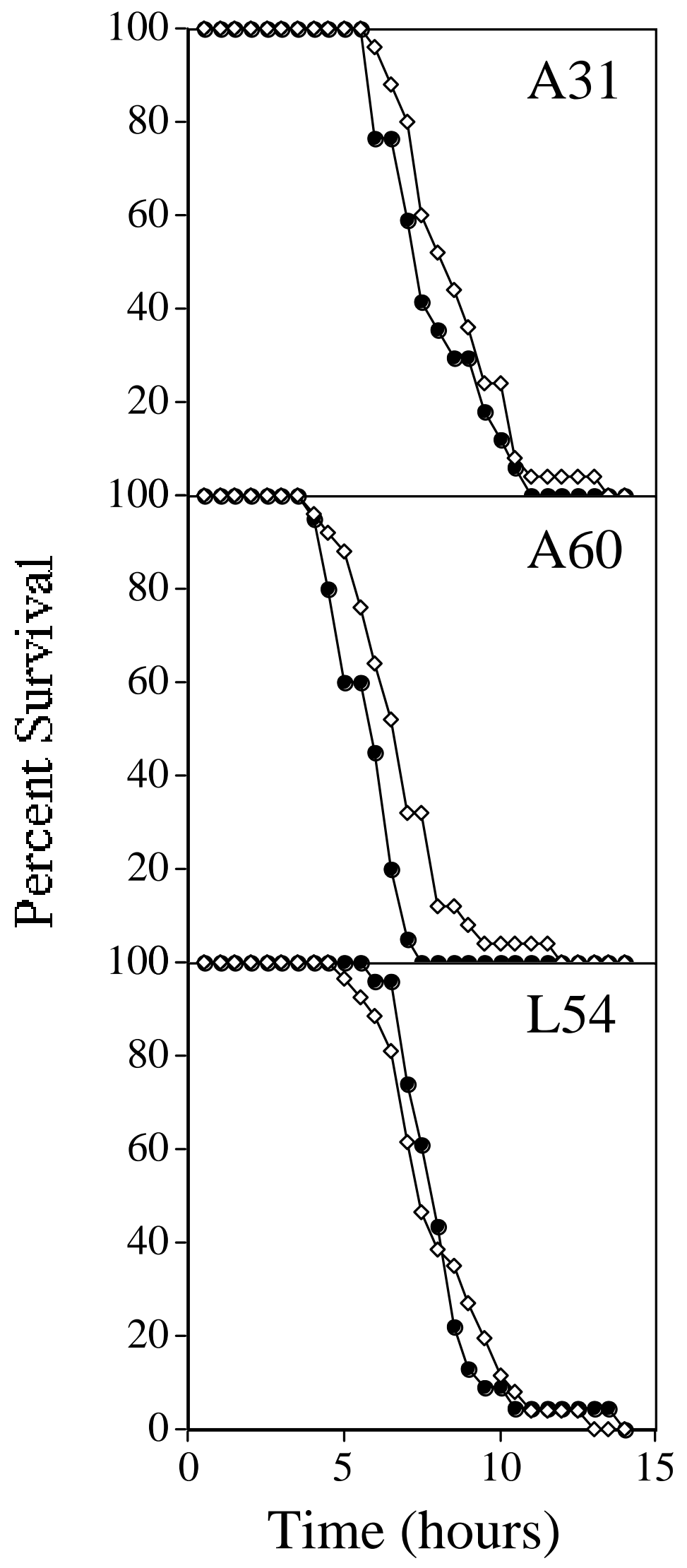

